\title{
On Sufficient Conditions for the Existence of Past-Present-Future Dependent Fixed Point in the Razumikhin Class and Application
}

\author{
Marwan Amin Kutbi ${ }^{1}$ and Wutiphol Sintunavarat ${ }^{2}$ \\ ${ }^{1}$ Department of Mathematics, King AbdulAziz University, P.O. Box 80203, Jeddah 21589, Saudi Arabia \\ ${ }^{2}$ Department of Mathematics and Statistics, Faculty of Science and Technology, Thammasat University Rangsit Center, \\ Pathumthani 12121, Thailand
}

Correspondence should be addressed to Wutiphol Sintunavarat; wutiphol@mathstat.sci.tu.ac.th

Received 17 September 2013; Revised 19 December 2013; Accepted 20 December 2013; Published 5 February 2014

Academic Editor: Hichem Ben-El-Mechaiekh

Copyright (c) 2014 M. A. Kutbi and W. Sintunavarat. This is an open access article distributed under the Creative Commons Attribution License, which permits unrestricted use, distribution, and reproduction in any medium, provided the original work is properly cited.

We introduce the new type of nonself mapping and study sufficient conditions for the existence of past-present-future (for short PPF) dependent fixed point for such mapping in the Razumikhin class. Also, we apply our result to prove the PPF dependent coincidence point theorems. Finally, we use PPF dependence techniques to obtain solution for a nonlinear integral problem with delay.

\section{Introduction}

It is well known that many problems in many branches of mathematics, such as optimization problems, equilibrium problems, and variational problems, can be transformed to fixed point problem of the form $T x=x$ for self-mapping $T$ defined on framework of metric space $(X, d)$ or vector space $(X,\|\cdot\|)$. Therefore, the applications of fixed point theory are very important in diverse disciplines of mathematics. The famous Banach's contraction mapping principle is one of the cornerstones in the development of fixed point theory. From inspiration of this work, several researchers heavily studied this field. For example, see works of Kannan [1], Chatterjea [2], Berinde [3], Cirić [4], Geraghty [5], Meir and Keeler [6], Suzuki [7], Mizogushi and Takahashi [8], Dass and Gupta [9], Jaggi [10], Lou [11], and so forth.

On the other hand, Bernfeld et al. [12] introduced the concept of Past-Present-Future (for short PPF) dependent fixed point or the fixed point with PPF dependence which is one type of fixed points for mappings that have different domains and ranges. They also established the existence of PPF dependent fixed point theorems in the Razumikhin class for
Banach type contraction mappings. These results are useful for proving the solutions of nonlinear functional differential and integral equations which may depend upon the past history, present data, and future consideration. The generalizations of this result have been investigated heavily by many mathematicians (see [13-18] and references therein).

In this paper, we will introduce the new type of nonself mapping called Ciric-rational type contraction mapping. Also, we will study the sufficient conditions for the existence of PPF dependent fixed point theorems for such mapping in Razumikhin class. Furthermore, we apply the main result to the existence of PPF dependence coincidence point theorems. In the last section, an application to an integral problem with delay is also given.

\section{Preliminaries}

In this section, we recall some concepts and definitions that will be required in the sequel. Throughout this paper, let $E$ denote a Banach space with the norm $\|\cdot\|_{E}, I$ denote a closed interval $[a, b]$ in $\mathbb{R}$, and $E_{0}=C(I, E)$ denote the set of all 
continuous $E$-valued functions on $I$ equips with the supremum norm $\|\cdot\|_{E_{0}}$ defined by

$$
\|\phi\|_{E_{0}}=\sup _{t \in I}\|\phi(t)\|_{E} .
$$

A point $\phi \in E_{0}$ is said to be a PPF dependent fixed point or a fixed point with PPF dependence of a nonself mapping $T: E_{0} \rightarrow E$ if $T \phi=\phi(c)$ for some $c \in I$.

For a fixed element $c \in I$, the Razumikhin or minimal class of functions in $E_{0}$ is defined by

$$
\mathscr{R}_{c}:=\left\{\phi \in E_{0}:\|\phi\|_{E_{0}}=\|\phi(c)\|_{E}\right\}
$$

It is easy to see that constant functions are member of $\mathscr{R}_{c}$.

The class $\mathscr{R}_{c}$ is algebraically closed with respect to difference if $\phi-\xi \in \mathscr{R}_{c}$ whenever $\phi, \xi \in \mathscr{R}_{c}$. Similarly, $\mathscr{R}_{c}$ is topologically closed if it is closed with respect to the topology on $E_{0}$ generated by the norm $\|\cdot\|_{E_{0}}$.

Definition 1 (see Bernfeld et al. [12]). The mapping $T: E_{0} \rightarrow$ $E$ is said to be Banach type contraction if there exists a real number $\alpha \in[0,1)$ such that

$$
\|T \phi-T \xi\|_{E} \leq \alpha\|\phi-\xi\|_{E_{0}}
$$

for all $\phi, \xi \in E_{0}$.

The following PPF dependent fixed point theorem is proved by Bernfeld et al. [12].

Theorem 2 (see Bernfeld et al. [12]). Let $T: E_{0} \rightarrow E$ be a Banach type contraction. If $\mathscr{R}_{c}$ is topologically closed and algebraically closed with respect to difference, then $T$ has a unique PPF dependent fixed point in $\mathscr{R}_{c}$.

\section{PPF Dependent Fixed Point Theorems}

In this section, we introduce the concept of the Ciricrational type contraction mappings. Also, we study sufficient condition for the existence of PPF dependent fixed point for such mapping.

Definition 3. The mapping $T: E_{0} \rightarrow E$ is called Ciric-rational type contraction if there exist real numbers $\alpha, \beta, \gamma, \delta, \kappa \in[0,1)$ with $\alpha+\beta+\gamma+2 \delta+\kappa<1$ and $c \in I$ such that

$$
\begin{gathered}
\|T \phi-T \xi\|_{E} \\
\leq \alpha \max \left\{\|\phi-\xi\|_{E_{0}},\|\phi(c)-T \phi\|_{E}\|\xi(c)-T \xi\|_{E},\right. \\
\left.\frac{\|\phi(c)-T \xi\|_{E}+\|\xi(c)-T \phi\|_{E}}{2}\right\} \\
+\frac{\beta\|\phi(c)-T \phi\|_{E}\|\xi(c)-T \xi\|_{E}}{1+\|\phi-\xi\|_{E_{0}}} \\
+\frac{\gamma\|\phi(c)-T \xi\|_{E}\|\xi(c)-T \phi\|_{E}}{1+\|\phi-\xi\|_{E_{0}}}
\end{gathered}
$$

$$
\begin{aligned}
& +\frac{\delta\|\phi(c)-T \phi\|_{E}\|\phi(c)-T \xi\|_{E}}{1+\|\phi-\xi\|_{E_{0}}} \\
& +\frac{\kappa\|\xi(c)-T \xi\|_{E}\|\xi(c)-T \phi\|_{E}}{1+\|\phi-\xi\|_{E_{0}}}
\end{aligned}
$$

for all $\phi, \xi \in E_{0}$.

Remark 4. (i) All Banach type, Kannan type, and Chatterjea type mappings are Ciric-rational type contraction mapping.

(ii) If $\beta=\gamma=\delta=\kappa=0$, then Ciric-rational type contraction mapping reduces to Ciric-type contraction.

(iii) If $\alpha=0$, then $T$ is a generalization and improvement of rational type contraction mapping.

Here, we prove PPF dependent fixed point theorems for Ciric-rational type contraction mappings.

Theorem 5. Let $T: E_{0} \rightarrow E$ be a Ciric-rational type contraction mapping. If $\mathscr{R}_{c}$ is topologically closed and algebraically closed with respect to difference, then $T$ has a unique PPF dependent fixed point in $\mathscr{R}_{c}$.

Moreover, for a fixed $\phi_{0} \in \mathscr{R}_{c}$, if a sequence $\left\{\phi_{n}\right\}$ of iterates of $T$ in $\mathscr{R}_{c}$ is defined by

$$
T \phi_{n-1}=\phi_{n}(c)
$$

for all $n \in \mathbb{N}$, then $\left\{\phi_{n}\right\}$ converges to a PPF dependent fixed point of $T$ in $\mathscr{R}_{c}$.

Proof. Let $\phi_{0}$ be an arbitrary function in $\mathscr{R}_{c} \subseteq E_{0}$. Since $T \phi_{0} \in E$, there exists $x_{1} \in E$ such that $T \phi_{0}=x_{1}$. Choose $\phi_{1} \in \mathscr{R}_{c}$ such that

$$
x_{1}=\phi_{1}(c)
$$

Since $\phi_{1} \in \mathscr{R}_{c} \subseteq E_{0}$ and by hypothesis, we get $T \phi_{1} \in E$. This implies that there exists $x_{2} \in E$ such that $T \phi_{1}=x_{2}$. Thus, we can choose $\phi_{2} \in \mathscr{R}_{c}$ such that

$$
x_{2}=\phi_{2}(c)
$$

By continuing this process, we can construct the sequence $\left\{\phi_{n}\right\}$ in $\mathscr{R}_{c} \subseteq E_{0}$ such that

$$
T \phi_{n-1}=\phi_{n}(c)
$$

for all $n \in \mathbb{N}$. Since $\mathscr{R}_{c}$ is algebraically closed with respect to difference, we have

$$
\left\|\phi_{n-1}-\phi_{n}\right\|_{E_{0}}=\left\|\phi_{n-1}(c)-\phi_{n}(c)\right\|_{E}
$$

for all $n \in \mathbb{N}$.

Next, we will show that $\left\{\phi_{n}\right\}$ is a Cauchy sequence in $\mathscr{R}_{c}$. 
For each $n \in \mathbb{N}$, we have

$$
\begin{aligned}
\left\|\phi_{n}-\phi_{n+1}\right\|_{E_{0}} & \\
= & \left\|\phi_{n}(c)-\phi_{n+1}(c)\right\|_{E} \\
= & \left\|T \phi_{n-1}-T \phi_{n}\right\|_{E} \\
\leq \alpha \max & \left\{\left\|\phi_{n-1}-\phi_{n}\right\|_{E_{0}},\left\|\phi_{n-1}(c)-T \phi_{n-1}\right\|_{E},\right. \\
& \left\|\phi_{n}(c)-T \phi_{n}\right\|_{E}, \\
& \left.\frac{\left\|\phi_{n-1}(c)-T \phi_{n}\right\|_{E}+\left\|\phi_{n}(c)-T \phi_{n-1}\right\|_{E}}{2}\right\}
\end{aligned}
$$$$
+\frac{\beta\left\|\phi_{n-1}(c)-T \phi_{n-1}\right\|_{E}\left\|\phi_{n}(c)-T \phi_{n}\right\|_{E}}{1+\left\|\phi_{n-1}-\phi_{n}\right\|_{E_{0}}}
$$$$
+\frac{\gamma\left\|\phi_{n-1}(c)-T \phi_{n}\right\|_{E}\left\|\phi_{n}(c)-T \phi_{n-1}\right\|_{E}}{1+\left\|\phi_{n-1}-\phi_{n}\right\|_{E_{0}}}
$$$$
+\frac{\delta\left\|\phi_{n-1}(c)-T \phi_{n-1}\right\|_{E}\left\|\phi_{n-1}(c)-T \phi_{n}\right\|_{E}}{1+\left\|\phi_{n-1}-\phi_{n}\right\|_{E_{0}}}
$$$$
+\frac{\kappa\left\|\phi_{n}(c)-T \phi_{n}\right\|_{E}\left\|\phi_{n}(c)-T \phi_{n-1}\right\|_{E}}{1+\left\|\phi_{n-1}-\phi_{n}\right\|_{E_{0}}}
$$$$
=\alpha \max \left\{\left\|\phi_{n-1}-\phi_{n}\right\|_{E_{0}},\left\|\phi_{n-1}(c)-\phi_{n}(c)\right\|_{E}\right. \text {, }
$$$$
\left.\left\|\phi_{n}(c)-\phi_{n+1}(c)\right\|_{E}, \frac{\left\|\phi_{n-1}(c)-\phi_{n+1}(c)\right\|_{E}}{2}\right\}
$$$$
+\frac{\beta\left\|\phi_{n-1}(c)-\phi_{n}(c)\right\|_{E}\left\|\phi_{n}(c)-\phi_{n+1}(c)\right\|_{E}}{1+\left\|\phi_{n-1}-\phi_{n}\right\|_{E_{0}}}
$$$$
+\frac{\delta\left\|\phi_{n-1}(c)-\phi_{n}(c)\right\|_{E}\left\|\phi_{n-1}(c)-\phi_{n+1}(c)\right\|_{E}}{1+\left\|\phi_{n-1}-\phi_{n}\right\|_{E_{0}}}
$$$$
=\alpha \max \left\{\left\|\phi_{n-1}-\phi_{n}\right\|_{E_{0}},\left\|\phi_{n-1}(c)-\phi_{n}(c)\right\|_{E^{\prime}}\right.
$$

$$
\left.\left\|\phi_{n}(c)-\phi_{n+1}(c)\right\|_{E}, \frac{\left\|\phi_{n-1}(c)-\phi_{n+1}(c)\right\|_{E}}{2}\right\}
$$$$
+\beta\left\|\phi_{n-1}(c)-\phi_{n}(c)\right\|_{E}\left(\frac{\left\|\phi_{n-1}(c)-\phi_{n}(c)\right\|_{E}}{1+\left\|\phi_{n-1}(c)-\phi_{n}(c)\right\|_{E}}\right)
$$$$
+\delta\left\|\phi_{n-1}(c)-\phi_{n+1}(c)\right\|_{E}\left(\frac{\left\|\phi_{n-1}(c)-\phi_{n}(c)\right\|_{E}}{1+\left\|\phi_{n-1}(c)-\phi_{n}(c)\right\|_{E}}\right)
$$$$
\leq \alpha \max \left\{\left\|\phi_{n-1}(c)-\phi_{n}(c)\right\|_{E},\left\|\phi_{n}(c)-\phi_{n+1}(c)\right\|_{E}\right. \text {, }
$$

$$
\left.\frac{\left\|\phi_{n-1}(c)-\phi_{n}(c)\right\|_{E}+\left\|\phi_{n}(c)-\phi_{n+1}(c)\right\|_{E}}{2}\right\}
$$

$$
\begin{aligned}
& +\beta\left\|\phi_{n-1}(c)-\phi_{n}(c)\right\|_{E}+\delta\left\|\phi_{n-1}(c)-\phi_{n+1}(c)\right\|_{E} \\
\leq & \alpha \max \left\{\left\|\phi_{n-1}(c)-\phi_{n}(c)\right\|_{E},\left\|\phi_{n}(c)-\phi_{n+1}(c)\right\|_{E}\right\} \\
& +\beta\left\|\phi_{n-1}(c)-\phi_{n}(c)\right\|_{E}+\delta\left\|\phi_{n-1}(c)-\phi_{n+1}(c)\right\|_{E} \\
= & \alpha \max \left\{\left\|\phi_{n-1}-\phi_{n}\right\|_{E_{0}},\left\|\phi_{n}-\phi_{n+1}\right\|_{E_{0}}\right\} \\
& +\beta\left\|\phi_{n-1}-\phi_{n}\right\|_{E_{0}}+\delta\left\|\phi_{n-1}-\phi_{n+1}\right\|_{E_{0}} \\
\leq & \alpha \max \left\{\left\|\phi_{n-1}-\phi_{n}\right\|_{E_{0}},\left\|\phi_{n}-\phi_{n+1}\right\|_{E_{0}}\right\} \\
& +\beta\left\|\phi_{n-1}-\phi_{n}\right\|_{E_{0}}+\delta\left\|\phi_{n-1}-\phi_{n}\right\|_{E_{0}}+\delta\left\|\phi_{n}-\phi_{n+1}\right\|_{E_{0}} .
\end{aligned}
$$

For fixed $n \in \mathbb{N}$, if $\max \left\{\left\|\phi_{n-1}-\phi_{n}\right\|_{E_{0}},\left\|\phi_{n}-\phi_{n+1}\right\|_{E_{0}}\right\}=$ $\left\|\phi_{n-1}-\phi_{n}\right\|_{E_{0}}$, then we get

$$
\begin{aligned}
\left\|\phi_{n}-\phi_{n+1}\right\|_{E_{0}} \leq & \alpha\left\|\phi_{n-1}-\phi_{n}\right\|_{E_{0}}+\beta\left\|\phi_{n-1}-\phi_{n}\right\|_{E_{0}} \\
& +\delta\left\|\phi_{n-1}-\phi_{n}\right\|_{E_{0}}+\delta\left\|\phi_{n}-\phi_{n+1}\right\|_{E_{0}} .
\end{aligned}
$$

This implies that

$$
\left\|\phi_{n}-\phi_{n+1}\right\|_{E_{0}} \leq\left(\frac{\alpha+\beta+\delta}{1-\delta}\right)\left\|\phi_{n-1}-\phi_{n}\right\|_{E_{0}} .
$$

On the other hand, if $\max \left\{\left\|\phi_{n-1}-\phi_{n}\right\|_{E_{0}},\left\|\phi_{n}-\phi_{n+1}\right\|_{E_{0}}\right\}=$ $\left\|\phi_{n}-\phi_{n+1}\right\|_{E_{0}}$, then we get

$$
\begin{aligned}
\left\|\phi_{n}-\phi_{n+1}\right\|_{E_{0}} \leq & \alpha\left\|\phi_{n}-\phi_{n+1}\right\|_{E_{0}}+\beta\left\|\phi_{n-1}-\phi_{n}\right\|_{E_{0}} \\
& +\delta\left\|\phi_{n-1}-\phi_{n}\right\|_{E_{0}}+\delta\left\|\phi_{n}-\phi_{n+1}\right\|_{E_{0}} .
\end{aligned}
$$

This implies that

$$
\left\|\phi_{n}-\phi_{n+1}\right\|_{E_{0}} \leq\left(\frac{\beta+\delta}{1-\alpha-\delta}\right)\left\|\phi_{n-1}-\phi_{n}\right\|_{E_{0}} .
$$

Now, we let

$$
k:=\max \left\{\frac{\alpha+\beta+\delta}{1-\delta}, \frac{\beta+\delta}{1-\alpha-\delta}\right\} .
$$

From (12) and (14), we get

$$
\left\|\phi_{n}-\phi_{n+1}\right\|_{E_{0}} \leq k\left\|\phi_{n-1}-\phi_{n}\right\|_{E_{0}}
$$

for all $n \in \mathbb{N}$. Repeated application of the above relation yields

$$
\left\|\phi_{n}-\phi_{n+1}\right\|_{E_{0}} \leq k^{n}\left\|\phi_{0}-\phi_{1}\right\|_{E_{0}}
$$

for all $n \in \mathbb{N}$.

For $m, n \in \mathbb{N}$ with $m>n$, we obtain that

$$
\begin{aligned}
\left\|\phi_{n}-\phi_{m}\right\|_{E_{0}} \leq & \left\|\phi_{n}-\phi_{n+1}\right\|_{E_{0}}+\left\|\phi_{n+1}-\phi_{n+2}\right\|_{E_{0}} \\
& +\cdots+\left\|\phi_{m-1}-\phi_{m}\right\|_{E_{0}} \\
\leq & \left(k^{n}+k^{n+1}+\cdots+k^{m-1}\right)\left\|\phi_{0}-\phi_{1}\right\|_{E_{0}} \\
\leq & \frac{k^{n}}{1-k}\left\|\phi_{0}-\phi_{1}\right\|_{E_{0}} .
\end{aligned}
$$


Since $0 \leq \alpha+\beta+\gamma+2 \delta+\kappa<1$, we have $0 \leq k<1$. This shows that the sequence $\left\{\phi_{n}\right\}$ is a Cauchy sequence in $\mathscr{R}_{c} \subseteq E_{0}$. By the completeness of $E_{0}$, we get $\left\{\phi_{n}\right\}$ converges to a limit point $\phi^{*} \in E_{0}$. Therefore, $\lim _{n \rightarrow \infty} \phi_{n}=\phi^{*}$; that is,

$$
\lim _{n \rightarrow \infty}\left\|\phi_{n}-\phi^{*}\right\|_{E_{0}}=0_{E_{0}}
$$

Further, since $\mathscr{R}_{c}$ is topologically closed, we have $\phi^{*} \in \mathscr{R}_{c}$ and thus

$$
\lim _{n \rightarrow \infty}\left\|\phi_{n}(c)-\phi^{*}(c)\right\|_{E}=0_{E}
$$

Now we prove that $\phi^{*}$ is a PPF dependent fixed point of $T$. From the assumption of Ciric-rational type contraction of $T$, we get

$$
\begin{aligned}
& \left\|T \phi^{*}-\phi^{*}(c)\right\|_{E} \\
& \leq\left\|T \phi^{*}-\phi_{n+1}(c)\right\|_{E}+\left\|\phi_{n+1}(c)-\phi^{*}(c)\right\|_{E} \\
& =\left\|T \phi^{*}-T \phi_{n}\right\|_{E}+\left\|\phi_{n+1}-\phi^{*}\right\|_{E_{0}} \\
& \leq \alpha \max \left\{\left\|\phi^{*}-\phi_{n}\right\|_{E_{0}},\left\|\phi^{*}(c)-T \phi^{*}\right\|_{E},\right. \\
& \left\|\phi_{n}(c)-T \phi_{n}\right\|_{E} \\
& \left.\frac{\left\|\phi^{*}(c)-T \phi_{n}\right\|_{E}+\left\|\phi_{n}(c)-T \phi^{*}\right\|_{E}}{2}\right\} \\
& +\frac{\beta\left\|\phi^{*}(c)-T \phi^{*}\right\|_{E}\left\|\phi_{n}(c)-T \phi_{n}\right\|_{E}}{1+\left\|\phi^{*}-\phi_{n}\right\|_{E_{0}}} \\
& +\frac{\gamma\left\|\phi^{*}(c)-T \phi_{n}\right\|_{E}\left\|\phi_{n}(c)-T \phi^{*}\right\|_{E}}{1+\left\|\phi^{*}-\phi_{n}\right\|_{E_{0}}} \\
& +\frac{\delta\left\|\phi^{*}(c)-T \phi^{*}\right\|_{E}\left\|\phi^{*}(c)-T \phi_{n}\right\|_{E}}{1+\left\|\phi^{*}-\phi_{n}\right\|_{E_{0}}} \\
& +\frac{\kappa\left\|\phi_{n}(c)-T \phi_{n}\right\|_{E}\left\|\phi_{n}(c)-T \phi^{*}\right\|_{E}}{1+\left\|\phi^{*}-\phi_{n}\right\|_{E_{0}}} \\
& +\left\|\phi_{n+1}-\phi^{*}\right\|_{E_{0}} \\
& =\alpha \max \left\{\left\|\phi^{*}-\phi_{n}\right\|_{E_{0}},\left\|\phi^{*}(c)-T \phi^{*}\right\|_{E},\right. \\
& \left\|\phi_{n}(c)-\phi_{n+1}(c)\right\|_{E} \\
& \left.\frac{\left\|\phi^{*}(c)-\phi_{n+1}(c)\right\|_{E}+\left\|\phi_{n}(c)-T \phi^{*}\right\|_{E}}{2}\right\} \\
& +\frac{\beta\left\|\phi^{*}(c)-T \phi^{*}\right\|_{E}\left\|\phi_{n}(c)-\phi_{n+1}(c)\right\|_{E}}{1+\left\|\phi^{*}-\phi_{n}\right\|_{E_{0}}}
\end{aligned}
$$

$$
\begin{aligned}
& +\frac{\gamma\left\|\phi^{*}(c)-\phi_{n+1}(c)\right\|_{E}\left\|\phi_{n}(c)-T \phi^{*}\right\|_{E}}{1+\left\|\phi^{*}-\phi_{n}\right\|_{E_{0}}} \\
& +\frac{\delta\left\|\phi^{*}(c)-T \phi^{*}\right\|_{E}\left\|\phi^{*}(c)-\phi_{n+1}(c)\right\|_{E}}{1+\left\|\phi^{*}-\phi_{n}\right\|_{E_{0}}} \\
& +\frac{\kappa\left\|\phi_{n}(c)-\phi_{n+1}(c)\right\|_{E}\left\|\phi_{n}(c)-T \phi^{*}\right\|_{E}}{1+\left\|\phi^{*}-\phi_{n}\right\|_{E_{0}}} \\
& +\left\|\phi_{n+1}-\phi^{*}\right\|_{E_{0}}
\end{aligned}
$$

for all $n \in \mathbb{N}$. Taking the limit as $n \rightarrow \infty$ in the above inequality, by (19) and (20), we have

$$
\left\|T \phi^{*}-\phi^{*}(c)\right\|_{E} \leq \alpha\left\|T \phi^{*}-\phi^{*}(c)\right\|_{E} .
$$

This implies that

$$
\left\|T \phi^{*}-\phi^{*}(c)\right\|_{E}=0
$$

and then

$$
T \phi^{*}=\phi^{*}(c) .
$$

Therefore, $\phi^{*}$ is a PPF dependent fixed point of $T$ in $\mathscr{R}_{c}$.

Finally, we prove the uniqueness of PPF dependent fixed point of $T$ in $\mathscr{R}_{c}$. Let $\phi^{*}$ and $\xi^{*}$ be two PPF dependent fixed points of $T$ in $\mathscr{R}_{c}$. Therefore,

$$
\begin{aligned}
& \left\|\phi^{*}-\xi^{*}\right\|_{E_{0}} \\
& =\left\|\phi^{*}(c)-\xi^{*}(c)\right\|_{E} \\
& =\left\|T \phi^{*}-T \xi^{*}\right\|_{E} \\
& \leq \alpha \max \left\{\left\|\phi^{*}-\xi^{*}\right\|_{E_{0}},\left\|\phi^{*}(c)-T \phi^{*}\right\|_{E},\right. \\
& \left\|\xi^{*}(c)-T \xi^{*}\right\|_{E}, \\
& \left.\frac{\left\|\phi^{*}(c)-T \xi^{*}\right\|_{E}+\left\|\xi^{*}(c)-T \phi^{*}\right\|_{E}}{2}\right\} \\
& +\frac{\beta\left\|\phi^{*}(c)-T \phi^{*}\right\|_{E}\left\|\xi^{*}(c)-T \xi^{*}\right\|_{E}}{1+\left\|\phi^{*}-\xi^{*}\right\|_{E_{0}}} \\
& +\frac{\gamma\left\|\phi^{*}(c)-T \xi^{*}\right\|_{E}\left\|\xi^{*}(c)-T \phi^{*}\right\|_{E}}{1+\left\|\phi^{*}-\xi^{*}\right\|_{E_{0}}} \\
& +\frac{\delta\left\|\phi^{*}(c)-T \phi^{*}\right\|_{E}\left\|\phi^{*}(c)-T \xi^{*}\right\|_{E}}{1+\left\|\phi^{*}-\xi^{*}\right\|_{E_{0}}} \\
& +\frac{\kappa\left\|\xi^{*}(c)-T \xi^{*}\right\|_{E}\left\|\xi^{*}(c)-T \phi^{*}\right\|_{E}}{1+\left\|\phi^{*}-\xi^{*}\right\|_{E_{0}}} \\
& =\alpha \max \left\{\left\|\phi^{*}-\xi^{*}\right\|_{E_{0}}\right. \text {, } \\
& \left.\frac{\left\|\phi^{*}(c)-\xi^{*}(c)\right\|_{E}+\left\|\xi^{*}(c)-\phi^{*}(c)\right\|_{E}}{2}\right\}
\end{aligned}
$$




$$
\begin{aligned}
& +\frac{\gamma\left\|\phi^{*}(c)-T \xi^{*}\right\|_{E}\left\|\xi^{*}(c)-T \phi^{*}\right\|_{E}}{1+\left\|\phi^{*}-\xi^{*}\right\|_{E_{0}}} \\
= & \alpha \max \left\{\left\|\phi^{*}-\xi^{*}\right\|_{E_{0}}, \frac{\left\|\phi^{*}-\xi^{*}\right\|_{E_{0}}+\left\|\xi^{*}-\phi^{*}\right\|_{E_{0}}}{2}\right\} \\
& +\frac{\gamma\left\|\phi^{*}(c)-\xi^{*}(c)\right\|_{E}\left\|\xi^{*}(c)-\phi^{*}(c)\right\|_{E}}{1+\left\|\phi^{*}-\xi^{*}\right\|_{E_{0}}} \\
= & \alpha\left\|\phi^{*}-\xi^{*}\right\|_{E_{0}}+\frac{\gamma\left\|\phi^{*}-\xi^{*}\right\|_{E_{0}}\left\|\xi^{*}-\phi^{*}\right\|_{E_{0}}}{1+\left\|\phi^{*}-\xi^{*}\right\|_{E_{0}}} \\
= & \alpha\left\|\phi^{*}-\xi^{*}\right\|_{E_{0}}+\gamma\left\|\phi^{*}-\xi^{*}\right\|_{E_{0}}\left(\frac{\left\|\phi^{*}-\xi^{*}\right\|_{E_{0}}}{1+\left\|\phi^{*}-\xi^{*}\right\|_{E_{0}}}\right) \\
\leq & \alpha\left\|\phi^{*}-\xi^{*}\right\|_{E_{0}}+\gamma\left\|\phi^{*}-\xi^{*}\right\|_{E_{0}} .
\end{aligned}
$$

Since $0 \leq \alpha+\gamma<1$, we have $\left\|\phi^{*}-\xi^{*}\right\|_{E_{0}}=0$ and hence $\phi^{*}=\xi^{*}$. Therefore, $T$ has a unique PPF dependent fixed point in $\mathscr{R}_{c}$. This completes the proof.

Remark 6. If the Razumikhin class $\mathscr{R}_{c}$ is not topologically closed, then the limit of the sequence $\left\{\phi_{n}\right\}$ in Theorem 5 may be outside of $\mathscr{R}_{c}$. Therefore, a PPF dependent fixed point of $T$ may not be unique.

By applying Theorem 5, we obtain the following result.

Corollary 7. Let $T: E_{0} \rightarrow E$ be a nonself mapping and there exists a real number $\alpha \in[0,1)$ such that

$$
\|T \phi-T \xi\|_{E} \leq \alpha\|\phi-\xi\|_{E_{0}}
$$

for all $\phi, \xi \in E_{0}$.

If there exists $c \in I$ such that $\mathscr{R}_{c}$ is topologically closed and algebraically closed with respect to difference, then $T$ has a unique PPF dependent fixed point in $\mathscr{R}_{c}$.

Moreover, for a fixed $\phi_{0} \in \mathscr{R}_{c}$, if a sequence $\left\{\phi_{n}\right\}$ of iterates of $T$ in $\mathscr{R}_{c}$ is defined by

$$
T \phi_{n-1}=\phi_{n}(c)
$$

for all $n \in \mathbb{N}$, then $\left\{\phi_{n}\right\}$ converges to a PPF dependent fixed point of $T$ in $\mathscr{R}_{c}$.

If we set $\beta=\gamma=\delta=\kappa=0$ in Theorem 5, we get the PPF dependent fixed point result for Ciric-type contraction mapping.

Corollary 8. Let $T: E_{0} \rightarrow E$ be a nonself mapping and there exist real number $0 \leq \alpha<1$ and $c \in I$ such that

$$
\begin{gathered}
\|T \phi-T \xi\|_{E} \\
\leq \alpha \max \left\{\|\phi-\xi\|_{E_{0}},\|\phi(c)-T \phi\|_{E}\|\xi(c)-T \xi\|_{E},\right. \\
\left.\frac{\|\phi(c)-T \xi\|_{E}+\|\xi(c)-T \phi\|_{E}}{2}\right\}
\end{gathered}
$$

for all $\phi, \xi \in E_{0}$.
If $\mathscr{R}_{c}$ is topologically closed and algebraically closed with respect to difference, then $T$ has a unique PPF dependent fixed point in $\mathscr{R}_{c}$.

Moreover, for a fixed $\phi_{0} \in \mathscr{R}_{c}$, if a sequence $\left\{\phi_{n}\right\}$ of iterates of $T$ in $\mathscr{R}_{c}$ is defined by

$$
T \phi_{n-1}=\phi_{n}(c)
$$

for all $n \in \mathbb{N}$, then $\left\{\phi_{n}\right\}$ converges to a PPF dependent fixed point of $T$ in $\mathscr{R}_{c}$.

If we set $\alpha=0$ in Theorem 5, we get the PPF dependent fixed point result for generalized ratio type contraction mapping.

Corollary 9. Let $T: E_{0} \rightarrow E$ be a nonself mapping and there exist real numbers $\beta, \gamma, \delta, \kappa \in[0,1)$ with $\beta+\gamma+2 \delta+\kappa<1$ and $c \in I$ such that

$$
\begin{aligned}
\|T \phi-T \xi\|_{E} \leq & \frac{\beta\|\phi(c)-T \phi\|_{E}\|\xi(c)-T \xi\|_{E}}{1+\|\phi-\xi\|_{E_{0}}} \\
& +\frac{\gamma\|\phi(c)-T \xi\|_{E}\|\xi(c)-T \phi\|_{E}}{1+\|\phi-\xi\|_{E_{0}}} \\
& +\frac{\delta\|\phi(c)-T \phi\|_{E}\|\phi(c)-T \xi\|_{E}}{1+\|\phi-\xi\|_{E_{0}}} \\
& +\frac{\kappa\|\xi(c)-T \xi\|_{E}\|\xi(c)-T \phi\|_{E}}{1+\|\phi-\xi\|_{E_{0}}}
\end{aligned}
$$

for all $\phi, \xi \in E_{0}$.

If $\mathscr{R}_{c}$ is topologically closed and algebraically closed with respect to difference, then $T$ has a unique PPF dependent fixed point in $\mathscr{R}_{c}$.

Moreover, for a fixed $\phi_{0} \in \mathscr{R}_{c}$, if a sequence $\left\{\phi_{n}\right\}$ of iterates of $T$ in $\mathscr{R}_{c}$ is defined by

$$
T \phi_{n-1}=\phi_{n}(c)
$$

for all $n \in \mathbb{N}$, then $\left\{\phi_{n}\right\}$ converges to a PPF dependent fixed point of $T$ in $\mathscr{R}_{c}$.

\section{PPF Dependent Coincidence Point Theorems}

Definition 10. Let $T: E_{0} \rightarrow E$ and $S: E_{0} \rightarrow E_{0}$ be two nonself mappings. A point $\phi \in E_{0}$ is said to be a PPF dependent coincidence point or a coincidence point with PPF dependence of $T$ and $S$ if $T \phi=(S \phi)(c)$ for some $c \in I$.

Next, we introduce the condition of the Ciric-rational type contraction for a pair of two nonself mappings.

Definition 11. Let $T: E_{0} \rightarrow E$ and $S: E_{0} \rightarrow E_{0}$ be two nonself mappings. The ordered pair $(T, S)$ is said to satisfy the condition of Ciric-rational type contraction if there exist real 
numbers $\alpha, \beta, \gamma, \delta, \kappa \in[0,1)$ with $\alpha+\beta+\gamma+2 \delta+\kappa<1$ and $c \in I$ such that

$$
\begin{gathered}
\|T \phi-T \xi\|_{E} \\
\leq \alpha \max \left\{\|S \phi-S \xi\|_{E_{0}},\right. \\
\|(S \phi)(c)-T \phi\|_{E}\|(S \xi)(c)-T \xi\|_{E}, \\
\left.\frac{\|(S \phi)(c)-T \xi\|_{E}+\|(S \xi)(c)-T \phi\|_{E}}{2}\right\} \\
+\frac{\beta\|(S \phi)(c)-T \phi\|_{E}\|(S \xi)(c)-T \xi\|_{E}}{1+\|S \phi-S \xi\|_{E_{0}}} \\
+\frac{\gamma\|(S \phi)(c)-T \xi\|_{E}\|(S \xi)(c)-T \phi\|_{E}}{1+\|S \phi-S \xi\|_{E_{0}}} \\
+\frac{\delta\|(S \phi)(c)-T \phi\|_{E}\|(S \phi)(c)-T \xi\|_{E}}{1+\|S \phi-S \xi\|_{E_{0}}} \\
+\frac{\kappa\|(S \xi)(c)-T \xi\|_{E}\|(S \xi)(c)-T \phi\|_{E}}{1+\|S \phi-S \xi\|_{E_{0}}}
\end{gathered}
$$

for all $\phi, \xi \in E_{0}$.

Remark 12. It is easy to see that

$(T, S)$ satisfies the condition of Ciric-rational type contraction and $S$ is identity mapping

$$
\Downarrow
$$

$T$ is a Ciric-rational type contraction mapping.

Now, we apply our result to the previous section to the PPF dependent coincidence point theorem.

Theorem 13. Let $T: E_{0} \rightarrow E$ and $S: E_{0} \rightarrow E_{0}$ be two nonself mappings. Suppose that the following conditions hold:

$\left(\star_{1}\right)(T, S)$ satisfies the condition of Ciric-rational type contraction;

$$
\left(\star_{2}\right) S\left(\mathscr{R}_{c}\right) \subseteq \mathscr{R}_{c} \text {. }
$$

If $S\left(\mathscr{R}_{c}\right)$ is topologically closed and algebraically closed with respect to difference, then $T$ and $S$ have a PPF dependent coincidence point.

Proof. For self-mapping $S: E_{0} \rightarrow E_{0}$, it is well know that there exists $F_{0} \subset E_{0}$ such that $S\left(F_{0}\right)=S\left(E_{0}\right)$ and $\left.S\right|_{F_{0}}$ is oneto-one. Since

$$
T\left(F_{0}\right) \subseteq T\left(E_{0}\right) \subseteq E,
$$

we can define a nonself mapping $\mathscr{H}: S\left(F_{0}\right) \rightarrow E$ by

$$
\mathscr{H}(S \phi)=T \phi
$$

for all $\phi \in F_{0}$. Since $S_{F_{0}}$ is one-to-one mapping, we have $\mathscr{H}$ is well-defined.
By the condition of Ciric-rational type contraction of $(T, S)$ and the construction of $\mathscr{H}$, we get

$$
\begin{gathered}
\|\mathscr{H}(S \phi)-\mathscr{H}(S \xi)\|_{E} \\
\leq \alpha \max \left\{\|S \phi-S \xi\|_{E_{0}},\right. \\
\quad\|(S \phi)(c)-\mathscr{H}(S \phi)\|_{E}\|(S \xi)(c)-\mathscr{H}(S \xi)\|_{E}, \\
\left.\frac{\|(S \phi)(c)-\mathscr{H}(S \xi)\|_{E}+\|(S \xi)(c)-\mathscr{H}(S \phi)\|_{E}}{2}\right\} \\
+\frac{\beta\|(S \phi)(c)-\mathscr{H}(S \phi)\|_{E}\|(S \xi)(c)-\mathscr{H}(S \xi)\|_{E}}{1+\|S \phi-S \xi\|_{E_{0}}} \\
+\frac{\gamma\|(S \phi)(c)-\mathscr{H}(S \xi)\|_{E}\|(S \xi)(c)-\mathscr{H}(S \phi)\|_{E}}{1+\|S \phi-S \xi\|_{E_{0}}} \\
+\frac{\delta\|(S \phi)(c)-\mathscr{H}(S \phi)\|_{E}\|(S \phi)(c)-\mathscr{H}(S \xi)\|_{E}}{1+\|S \phi-S \xi\|_{E_{0}}} \\
+\frac{\kappa\|(S \xi)(c)-\mathscr{H}(S \xi)\|_{E}\|(S \xi)(c)-\mathscr{H}(S \phi)\|_{E}}{1+\|S \phi-S \xi\|_{E_{0}}}
\end{gathered}
$$

for all $S \phi, S \xi \in S\left(E_{0}\right)$. This implies that $\mathscr{H}$ is a Ciric-rational type contraction mapping.

Using Theorem 5 with a mapping $\mathscr{H}$, we can find a unique PPF dependent fixed point of $\mathscr{H}$. Let a unique PPF dependent fixed point of $\mathscr{H}$ be $\zeta \in S\left(F_{0}\right)$; that is, $\mathscr{H} \zeta=\zeta(c)$. Since $\zeta \in$ $S\left(F_{0}\right)$, we can find $\omega \in F_{0}$ such that $\zeta=S \omega$. Now, we have

$$
T \omega=\mathscr{H}(S \omega)=\mathscr{H} \zeta=\zeta(c)=(S \omega)(c)
$$

Therefore, $\omega$ is a PPF dependent coincidence point of $T$ and $S$. This completes the proof. ies.

By applying Theorem 13, we obtain the following corollar-

Corollary 14. Let $T: E_{0} \rightarrow E$ and $S: E_{0} \rightarrow E_{0}$ be two nonself mappings. Suppose that the following conditions hold:

$\left(\star_{1}\right)$ there exists a real number $\alpha \in[0,1)$ such that

$$
\|T \phi-T \xi\|_{E} \leq \alpha\|S \phi-S \xi\|_{E_{0}}
$$

for all $\phi, \xi \in E_{0}$;

$\left(\star_{2}\right)$ there exists $c \in I$ such that $S\left(\mathscr{R}_{c}\right) \subseteq \mathscr{R}_{c}$.

If $S\left(\mathscr{R}_{c}\right)$ is topologically closed and algebraically closed with respect to difference, then $T$ and $S$ have a PPF dependent coincidence point in $\mathscr{R}_{c}$.

Corollary 15. Let $T: E_{0} \rightarrow E$ and $S: E_{0} \rightarrow E_{0}$ be two nonself mappings. Suppose that the following conditions hold: 
$\left(\star_{1}\right)$ there exist real numbers $0 \leq \alpha<1$ and $c \in I$ such that

$$
\begin{aligned}
\|T \phi-T \xi\|_{E} & \\
\leq \alpha \max & \left\{\|S \phi-S \xi\|_{E_{0}},\right. \\
& \|(S \phi)(c)-T \phi\|_{E}\|(S \xi)(c)-T \xi\|_{E}, \\
& \left.\frac{\|(S \phi)(c)-T \xi\|_{E}+\|(S \xi)(c)-T \phi\|_{E}}{2}\right\}
\end{aligned}
$$

$$
\begin{aligned}
& \text { for all } \phi, \xi \in E_{0} ; \\
& \left(\star_{2}\right) S\left(\mathscr{R}_{c}\right) \subseteq \mathscr{R}_{c} .
\end{aligned}
$$

If $S\left(\mathscr{R}_{c}\right)$ is topologically closed and algebraically closed with respect to difference, then $T$ and $S$ have a PPF dependent coincidence point in $\mathscr{R}_{c}$.

Corollary 16. Let $T: E_{0} \rightarrow E$ and $S: E_{0} \rightarrow E_{0}$ be two nonself mappings. Suppose that the following conditions hold:

$\left(\star_{1}\right)$ there exist real numbers $\beta, \gamma, \delta, \kappa \in[0,1)$ with $\beta+$ $\gamma+2 \delta+\kappa<1$ and $c \in I$ such that

$$
\begin{aligned}
\|T \phi-T \xi\|_{E} \leq & +\frac{\beta\|(S \phi)(c)-T \phi\|_{E}\|(S \xi)(c)-T \xi\|_{E}}{1+\|S \phi-S \xi\|_{E_{0}}} \\
& +\frac{\gamma\|(S \phi)(c)-T \xi\|_{E}\|(S \xi)(c)-T \phi\|_{E}}{1+\|S \phi-S \xi\|_{E_{0}}} \\
& +\frac{\delta\|(S \phi)(c)-T \phi\|_{E}\|(S \phi)(c)-T \xi\|_{E}}{1+\|S \phi-S \xi\|_{E_{0}}} \\
& +\frac{\kappa\|(S \xi)(c)-T \xi\|_{E}\|(S \xi)(c)-T \phi\|_{E}}{1+\|S \phi-S \xi\|_{E_{0}}}
\end{aligned}
$$

for all $\phi, \xi \in E_{0}$;

$$
\left(\star_{2}\right) S\left(\mathscr{R}_{c}\right) \subseteq \mathscr{R}_{c} \text {. }
$$

If $S\left(\mathscr{R}_{c}\right)$ is topologically closed and algebraically closed with respect to difference, then $T$ and $S$ have a PPF dependent coincidence point in $\mathscr{R}_{c}$.

\section{Application to a Nonlinear Integral Equation}

In this section, we apply our result to study the existence and uniqueness of solution of a nonlinear integral equation.

Given a closed interval $J:=[j, 0]$ such that $j \in \mathbb{R}^{-}$, let $\Omega_{0}$ denote the space of continuous real-valued functions defined on $J$. We equip the space $\Omega_{0}$ with supremum normed $\|\cdot\|_{\Omega_{0}}$ defined by

$$
\|\phi\|_{\Omega_{0}}=\sup _{t \in J}|\phi(t)|
$$

It well known that $\Omega_{0}$ is a Banach space with this normed.
For fixed $T \in \mathbb{R}^{+}$, for each $t \in I:=[0, T]$, define a function $t \mapsto \phi_{t}$ by

$$
\phi_{t}(a)=\phi(t+a), \quad \text { for } a \in J,
$$

where the argument $a$ represents the delay in the argument of solutions.

Given $\varsigma \in C(I, \mathbb{R})$, we will consider the following nonlinear integral problem:

$$
\phi(t)=\varsigma(0)+\int_{0}^{T} G(T, s) f\left(s, \phi_{s}\right) d s
$$

for all $t \in I$, where $\phi \in C(I, \mathbb{R}), f \in C(I \times C(J, \mathbb{R}), \mathbb{R})$, and $G \in C\left(I \times I, \mathbb{R}_{+}\right)$.

Theorem 17. Problem (42) has only one solution defined on $J \cup I$ if the following conditions hold:

$$
\left(\nabla_{1}\right) \sup _{t \in I}\left(\int_{0}^{t} G(t, s) d s\right) \leq 1
$$

$\left(\nabla_{2}\right)$ there exist nonnegative real number $\alpha<1$ such that, for all $t \in I$ and $\phi, \xi \in C(I, \mathbb{R})$, one has

$$
|f(t, \phi)-f(t, \xi)| \leq \alpha|\phi(0)-\xi(0)| \text {. }
$$

Proof. Define the following set:

$$
\widehat{E}:=\left\{\widehat{\phi}=\left(\phi_{t}\right)_{t \in I}: \phi_{t} \in \Omega_{0}, \phi \in C(I, \mathbb{R})\right\} .
$$

Also, define the normed $\|\cdot\|_{\widehat{E}}$ in $\widehat{E}$ by

$$
\|\widehat{\phi}\|_{\widehat{E}}:=\sup _{t \in I}\left\|\phi_{t}\right\|_{\Omega_{0}}
$$

We obtain that $\widehat{\phi} \in C(J, \mathbb{R})$. Next, we show that $\widehat{E}$ is complete. Consider a Cauchy sequence $\left\{\widehat{\phi}_{n}\right\}$ in $\widehat{E}$. It is easy to see that $\left\{\phi_{n_{t}}\right\}_{t \in I}$ is a Cauchy sequence in $C(J, \mathbb{R})$ for all $t \in I$. This implies that $\left\{\phi_{n_{t}}(s)\right\}$ is a Cauchy sequence in $\mathbb{R}$ for each $s \in J$. So $\phi_{n_{t}}(s)$ converges to $\phi_{t}(s)$ for each $s \in J$. Since $\left\{\phi_{n_{t}}\right\}$ is a sequence of uniformly continuous functions for a fixed $t \in I$, $\phi_{t}(s)$ is also continuous in $s \in J$. Thus going backwards we get that $\widehat{\phi}_{n}$ converges to $\widehat{\phi}$ in $\widehat{E}$. Therefore, $\widehat{E}$ is complete.

Next, we define the function $T: \widehat{E} \rightarrow \mathbb{R}$ by

$$
T \widehat{\phi} \equiv T\left(\phi_{t}\right)_{t \in I}:=\varsigma(0)+\int_{0}^{T} G(T, s) f\left(s, \phi_{s}\right) d s .
$$


For $\widehat{\phi}, \widehat{\xi} \in \widehat{E}$, we have

$$
\begin{aligned}
& |T \widehat{\phi}-T \widehat{\xi}|=\left|\int_{0}^{T} G(T, s) f\left(s, \phi_{s}\right) d s-\int_{0}^{T} G(T, s) f\left(s, \xi_{s}\right) d s\right| \\
& =\left|\int_{0}^{T}\left(G(T, s) f\left(s, \phi_{s}\right)-G(T, s) f\left(s, \xi_{s}\right)\right) d s\right| \\
& =\int_{0}^{T}\left(G(T, s) f\left(s, \phi_{s}\right)-G(T, s) f\left(s, \xi_{s}\right)\right) d s \\
& \leq \int_{0}^{T}\left|G(T, s) f\left(s, \phi_{s}\right)-G(T, s) f\left(s, \xi_{s}\right)\right| d s \\
& =\int_{0}^{T} G(T, s)\left|f\left(s, \phi_{s}\right)-f\left(s, \xi_{s}\right)\right| d s \\
& \leq \int_{0}^{T} G(T, s) \alpha\left|\phi_{s}(0)-\xi_{s}(0)\right| d s \\
& =\int_{0}^{T} G(T, s) \alpha|\phi(s)-\xi(s)| d s \\
& \leq \int_{0}^{T} G(T, s) \alpha\|\widehat{\phi}-\widehat{\xi}\|_{\widehat{E}} d s \\
& =\alpha\|\widehat{\phi}-\widehat{\xi}\|_{\widehat{E}}\left(\int_{0}^{T} G(T, s) d s\right) \\
& \leq \alpha\|\widehat{\phi}-\widehat{\xi}\|_{\widehat{E}}\left[\sup _{t \in I}\left(\int_{0}^{t} G(t, s) d s\right)\right] \\
& \leq \alpha\|\widehat{\phi}-\widehat{\xi}\|_{\widehat{E}} \text {. }
\end{aligned}
$$

This implies that $T$ is a Ciric-rational type contraction.

Moreover, the Razumikhin $\mathscr{R}_{0}$ is $C(I, \mathbb{R})$ which is topologically closed and algebraically closed with respect to difference. Now all hypotheses of Theorem 5 are automatically satisfied with $c=0$. Therefore, there exists PPF dependence fixed point $\widehat{\phi}^{*}$ of $T$; that is, $T \widehat{\phi}^{*}=\widehat{\phi}^{*}(0)$. This implies that

$$
\varsigma(0)+\int_{0}^{T} G(T, s) f\left(s, \widehat{\phi}_{s}^{*}\right) d s=\left(\widehat{\phi}_{t}^{*}(0)\right)_{t \in I}=\left(\widehat{\phi}^{*}(t)\right)_{t \in I}
$$

Hence, the integral equation (42) has a solution. This completes the proof.

\section{Conflict of Interests}

The authors declare that they have no competing interests.

\section{Acknowledgment}

The first author gratefully acknowledges the support from the Deanship of Scientific Research (DSR) at King Abdulaziz University (KAU) during this research.

\section{References}

[1] R. Kannan, "Some results on fixed points-II," The American Mathematical Monthly, vol. 76, pp. 405-408, 1969.

[2] S. K. Chatterjea, "Fixed-point theorems," Comptes Rendus de l'Académie Bulgare des Sciences, vol. 25, pp. 727-730, 1972.

[3] V. Berinde, "Approximating fixed points of weak contractions using the Picard iteration," Nonlinear Analysis Forum, vol. 9, no. 1, pp. 43-53, 2004.

[4] L. B. Ćirić, "A generalization of Banach's contraction principle," Proceedings of the American Mathematical Society, vol. 45, pp. 267-273, 1974.

[5] M. A. Geraghty, "On contractive mappings," Proceedings of the American Mathematical Society, vol. 40, pp. 604-608, 1973.

[6] A. Meir and E. Keeler, "A theorem on contraction mappings," Journal of Mathematical Analysis and Applications, vol. 28, pp. 326-329, 1969.

[7] T. Suzuki, "A generalized Banach contraction principle that characterizes metric completeness," Proceedings of the American Mathematical Society, vol. 136, no. 5, pp. 1861-1869, 2008.

[8] N. Mizoguchi and W. Takahashi, "Fixed point theorems for multivalued mappings on complete metric spaces," Journal of Mathematical Analysis and Applications, vol. 141, no. 1, pp. 177$188,1989$.

[9] B. K. Dass and S. Gupta, "An extension of Banach contraction principle through rational expression," Indian Journal of Pure and Applied Mathematics, vol. 6, no. 12, pp. 1455-1458, 1975.

[10] D. S. Jaggi, "Some unique fixed point theorems," Indian Journal of Pure and Applied Mathematics, vol. 8, no. 2, pp. 223-230, 1977.

[11] B. Lou, "Fixed points for operators in a space of continuous functions and applications," Proceedings of the American Mathematical Society, vol. 127, no. 8, pp. 2259-2264, 1999.

[12] S. R. Bernfeld, V. Lakshmikantham, and Y. M. Reddy, "Fixed point theorems of operators with PPF dependence in Banach spaces," Applicable Analysis, vol. 6, no. 4, pp. 271-280, 1977.

[13] R. P. Agarwal, W. Sintunavarat, and P. Kumam, "PPF dependent fixed point theorems for an $\alpha_{c}$-admissible non-self mapping in the Razumikhin class," Fixed Point Theory and Applications, vol. 2013, article 280, 2013.

[14] B. C. Dhage, "On some common fixed point theorems with PPF dependence in Banach spaces," Journal of Nonlinear Science and Its Applications, vol. 5, no. 3, pp. 220-232, 2012.

[15] B. C. Dhage, "Fixed point theorems with PPF dependence and functional differential equations," Fixed Point Theory, vol. 13, no. 2, pp. 439-452, 2012.

[16] Z. Drici, F. A. McRae, and J. Vasundhara Devi, "Fixed-point theorems in partially ordered metric spaces for operators with PPF dependence," Nonlinear Analysis: Theory, Methods \& Applications A, vol. 67, no. 2, pp. 641-647, 2007.

[17] Z. Drici, F. A. McRae, and J. Vasundhara Devi, "Fixed point theorems for mixed monotone operators with PPF dependence," Nonlinear Analysis: Theory, Methods \& Applications A, vol. 69, no. 2, pp. 632-636, 2008.

[18] W. Sintunavarat and P. Kumam, "PPF dependent fixed point theorems for rational type contraction mappings in Banach spaces," Journal of Nonlinear Analysis and Optimization, vol. 4, no. 2, pp. 157-162, 2013. 


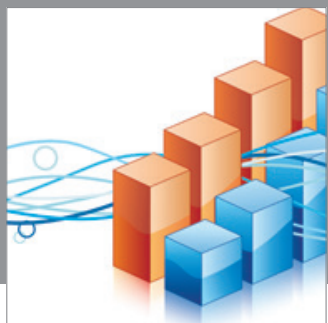

Advances in

Operations Research

mansans

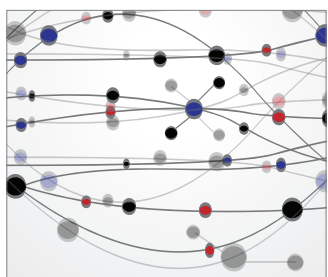

The Scientific World Journal
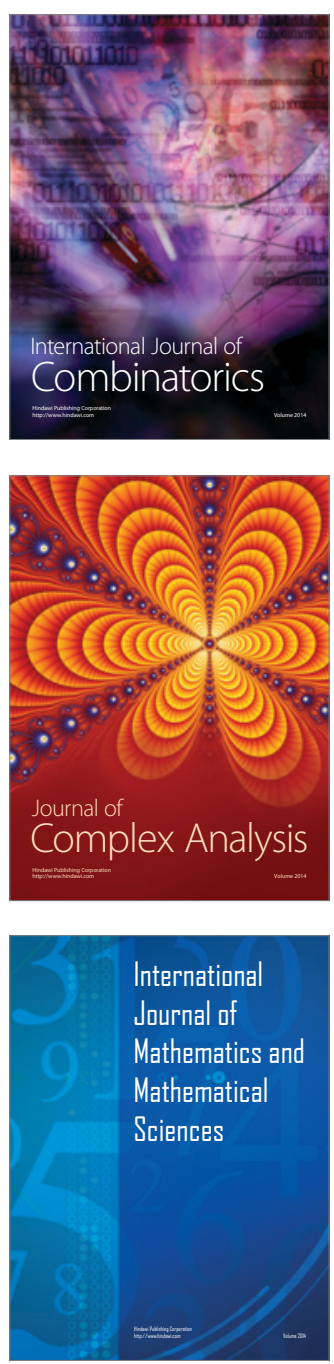
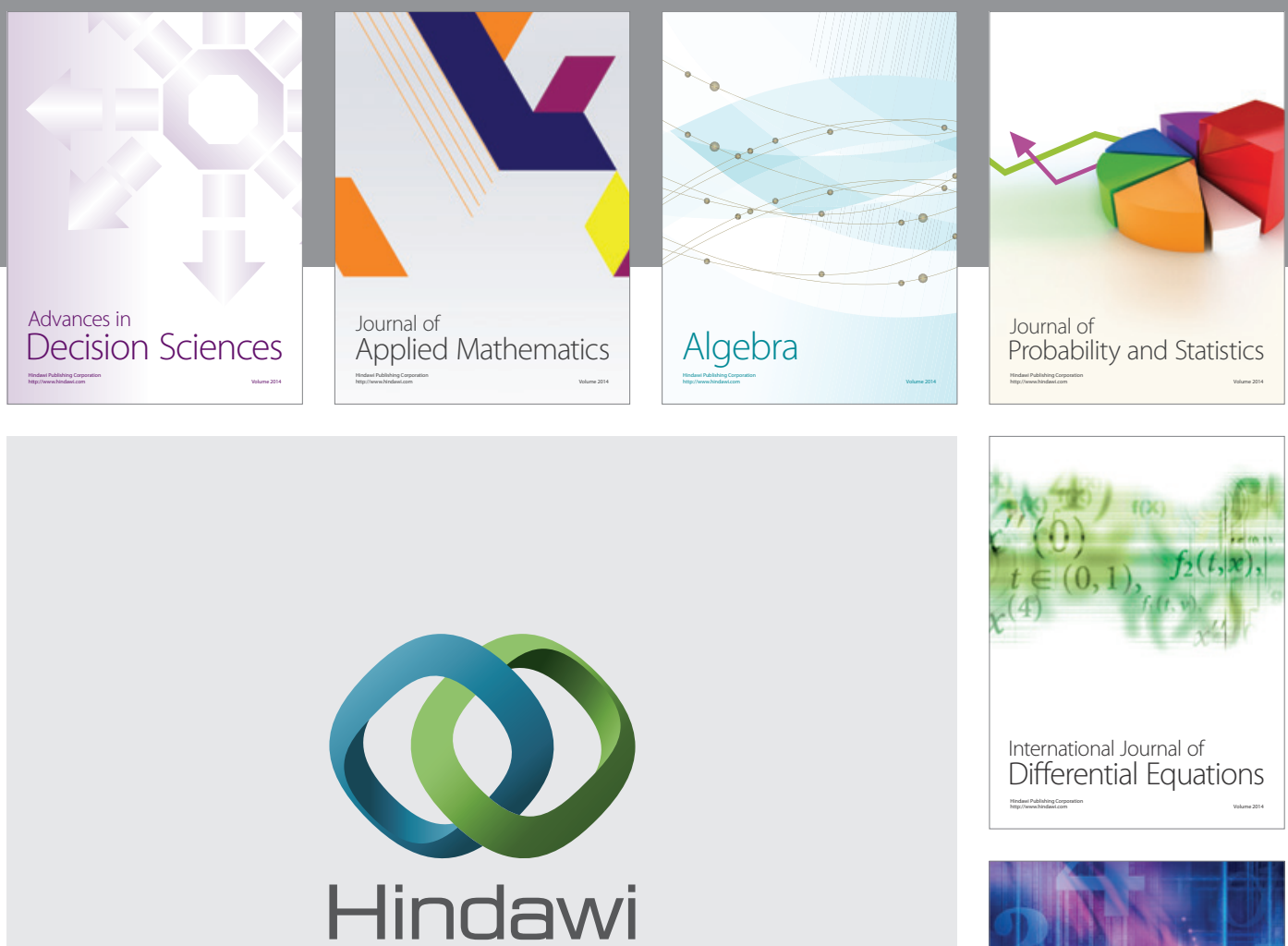

Submit your manuscripts at http://www.hindawi.com
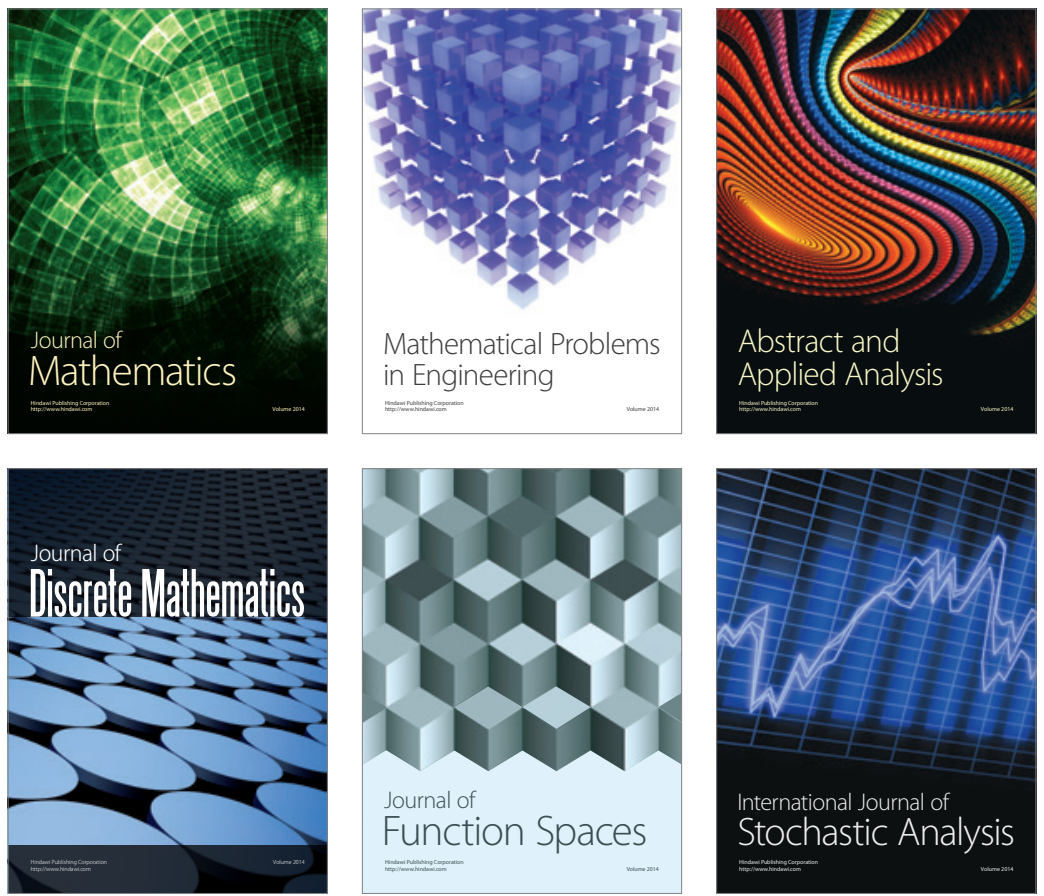

Journal of

Function Spaces

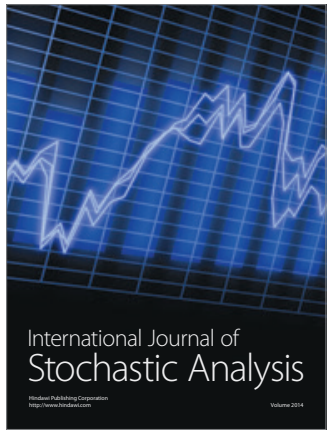

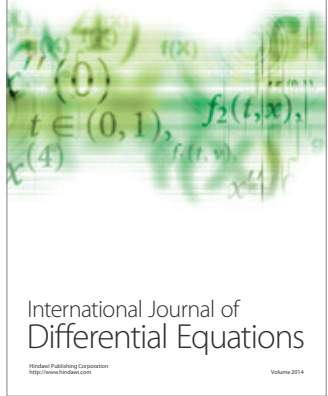
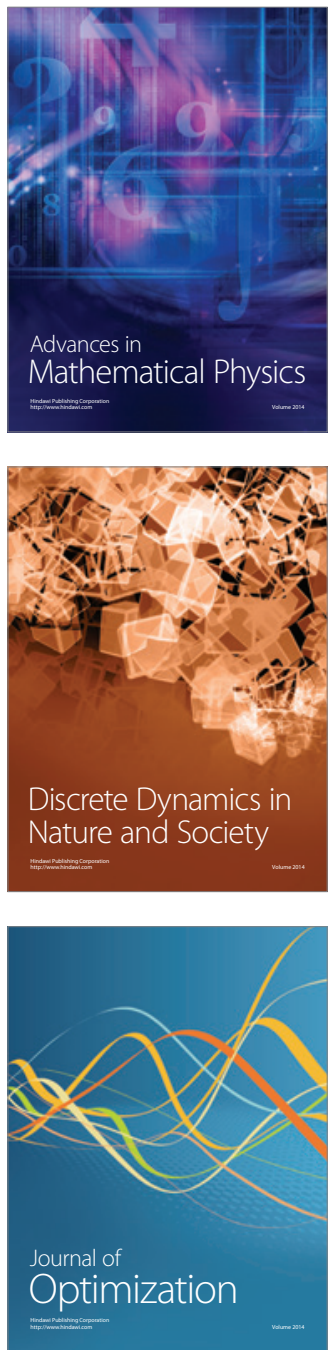